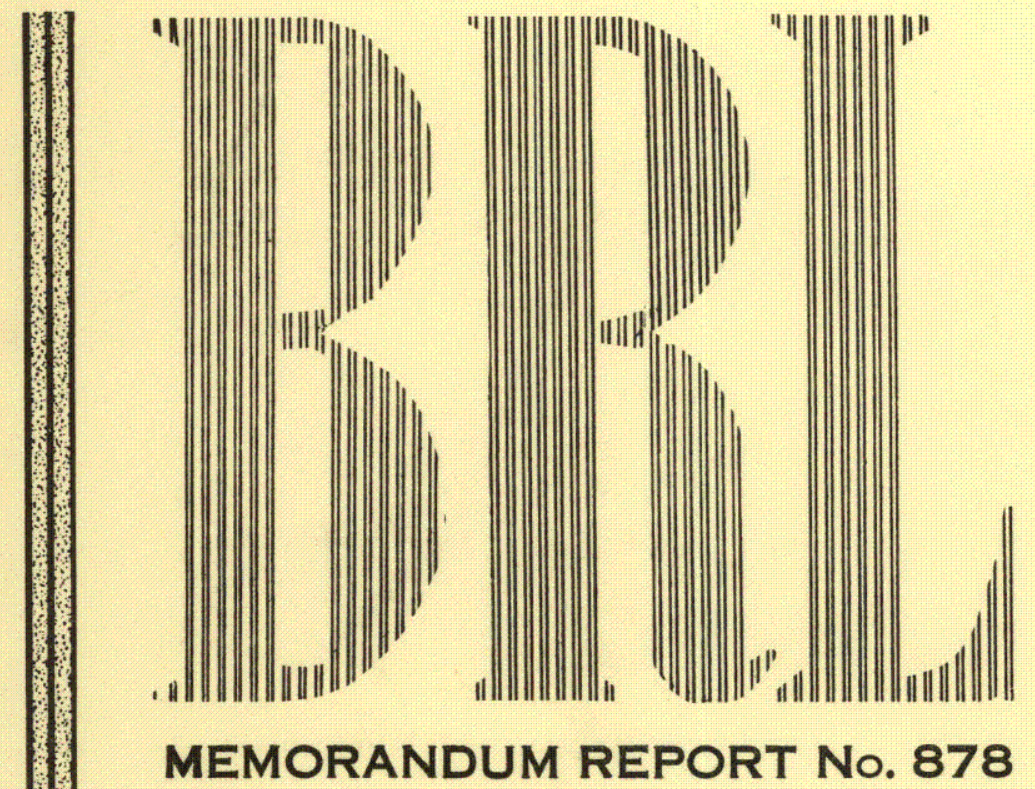

\title{
An Elementary Review \\ Of The Mathieu-Hill Equation \\ Of Real Variable \\ Based On Numerical Solutions
}

\section{S. J. ZAROODNY}

DEPARTMENT OF THE ARMY PROJECT No. 503-03-001

ORDNANCE RESEARCH AND DEVELOPMENT PROJECT NO. TB3-0108

BALLISTIC RESEARCH LABORATORIES 


\section{Destroy when no longer needed. DO NOT RETURN}




\author{
B A L I I T I C RESEARCH LABORATORIES \\ MEYORANDUM FEPORI NO. 878
}

AFRIL 1955 AN BLEMRNTARY HEVIEU OF THE MATHIEU-HILI EQUATION OF REAL VARIABLE
BASED ON NUMERICAL SOLUTIONS

5. J. Za roodny

Department of the Army Project No. 503-03-00I Ordnance Research and Development Project No. TB3-0108

ABERDEEN PROVING GROUND, MARYLAND 
TABLB OF CONTENTS

Page

INTRODUCTION .................... 5

PFRLIMINARIES .................. 6

NORMAL MODES ... ................ 7

FORMATION OF THE SBCOND SOLUTION ........... 9

PRACTICAL COMPUTATIONS. . . . . . . . . . . 9

CIASSIFICATION OF SOLUTIONS $\ldots \ldots$ IO

(a) Unstable Solutions ............ IO

(b) Stable Solutions ............. 12

(c) Cha racteristic Conditions ......... 12

(1) Characteristic Solutions ........ 23

(2) Secular Solutions ........... 16

PERIODIC KERNELS . . . . . . . . . 。 . 。 18

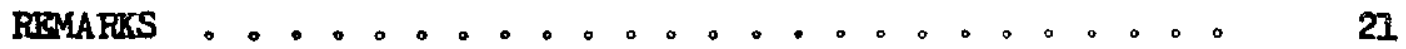

DESCRIPTION OP AVAILABLE TRAJECTORIES

DESCRIPITON OF THE CHAFT . . . . . . . . . . 24

IILUSTRATIONS:

Figure 1 Iso-So Chart ............ 27

Figure 2 Ince's Chart .......... 28

FRPERENCES .................. ... 29 


\author{
BALLISTIC RESEARCH LABORATORIES
}

MEMORANDUM MEPOFI NO. 878

\author{
SJZaroodny/plg \\ Aberdeen Proving Ground, Md. \\ April 1955
}

\title{
AN ELEMENTARY REVIEN OF THE MATHIEU-HILL EQUATION OF PRAL VARIABLE BASED ON NUMERICAL SOLUTIONS
}

\begin{abstract}
A description is given of a large number of trajectories of Mathieu's equation made on the ENIAC in 1948 and available at BRL. A large chart describing the behaviour of the solutions is given. The occasion is taken to review the essentials of the Mathieu theory for the benefit of the occasional user-proceeding from the point of view of an inspection of the numerical data.
\end{abstract}





\section{INTRODUCTION}

In a number of problems of mathematics and engineering there arises the Mathieu equation

$$
d^{2} y / d z^{2}+(a-2 q \cos 2 z) y=0
$$

where $a$ and $q$ are constants and the factor of 2 under the cosine symbol is use $\bar{d}$ largely for historical reasons. A number of alternative forms may convey the physical significance of a particular problem somewhat more clearly: $e_{\circ} g_{\circ}$, the periodic coefficient may be written as $n^{2}(1+k \cos 2 z)$, $\mathrm{N}^{2}(1+k \cos z),(1+k \cos N z)$, etco When generalized by substituting any periodic (usually even) function $J(z)$ for the cosine, the equation is called Hill's equation. The subject may be the outset divided in two parts: $z$ might be either real, or complex (e.g.g if $z$ is pure imaginary, this is equivalent to replacing $\cos$ by $\cos \mathrm{h}$ ). We shall here consider only the former case; then the subject is a generalization of simple harmonic motion and more particularly, of the theory of the vibrating string, though it differs from that simple theory considerably. Thus, in the theory of harmonic motion the only parameter (angular frequency for each mode) can be readily made $I$ by suitable choice of the scale of $z$; but when the "spring stiffness" term acquires the character of periodic oscillations, no fewer than two additional parameters must be considered (e.g, a and $q$, the scale of $\underline{z}$ having been fixed arbitrarily). The solutions exhibit a considerable variety; depending upon the combination of a and $q$ they may be stable, unstable or periodic: their description requires a description of the a-q plane, which may be divided into regions of stability and instability. (Cf。 Fig。2。). The Mathieu equation was introduced (in 1868) in the study of the vibrations of a membrane; it is the refore natural that these equations possess an eigenvalue character. This character is exhibited by a definite periodicity of certain solutions belonging to the border-lines between the stable and unstable regions in the a-q plane. In a number of problems, however, this elgenvalue character is not of the essence. The subject, therefore, may be further divided into two parts: the boundaryvalue problems ( $i . \theta .$, essentially the determination of those a and $q$ which would yield solutions of specified periodicity) and "what may be called initialovalue problems"。*

There exists an extensive literature on these eauations: and in particular, a wollonigh exhaustive compendium was given by McLachlan ${ }^{1}$ in 1947. Traditionally, the approach has been via the use of infinite series; as a result, the thorough study of the subject encompasses much auxiliary detail (such as recurrence relations, proofs and comparisons of convergence), the labor of which may easily distract and discourage a

Reference $I_{2}$ page 9

1 Superscripts refer to references. 
mere occasional user of Mathieu theory; while often ${ }_{2}$ formal solution .... is merely a steppingestone to quantitative resultg"n2. The modern growth of highospeed computing machinery (even though it should not be confused with mathematics) cannot help but exert some influence on the approach to the problems of this sort; thus, it should certainly de-emphasize the use of infinite series as a mere means to obtaining a solution of a differential equation. Thus, in 1948 Brillouin 3 emphasized that the numerical approach is particularly fruitful in dealing with Hill's equation. In fact, one of the earliest uses of ENIAC at the University of Pennsylvania was a systematized compilation of some 200 trajectories of the Mathieu equation by Brainerd et al4. The Ballistic Research Iaboratories ${ }^{5}$ had computed on the ENIAC at the Aberdeen Proving Ground some 1300 additional trajectories. These results contain a wealth of information; and it is the object of this report to present at least a small fraction of this information.

In introducing these numerical results it appearg that the necessary explanations can be easily extended to constituteg to an cccasional user of Mathieu theory, a rather self-sufficient exposition of the essentials of this theory: $i . \theta_{0}$ it can contain the principal proofs as well as an "empirical" description of the behaviour of the solutions, as fixed by the paremeters $a$ and $q$ and the inftial conditions. Since the method of solving a differential equation numerically, by its nature, represents the initialvalue approach, the eigenvalue character of the equation need not be presumed a priori, but arises naturally.

\section{PFELTMINARTRS}

The solutions of (1) possess two useful fundamental properties: they can be "translated" and "reflected".

Because of the periodicity of the coefficient in (I), if $y(z)$ is a solution, its translation by $\pi_{9} i_{0} \theta_{0}, y(z+\pi)$, is also a solution: for this represents merely a shift of the origin of $z$ by $\pi$, which leaves (I) unchanged. Note that a translation by $\pi / 2$, or a shift of the origin by $\pi / 2$, represents a change from $+q$ to $q, i_{0} e_{0}$, changes ( 1 ); and the refore cannot be allowed if a solution of (l) is sought.

The second property is brought out particularly clearly by the even form of the coefficient of $y$ in (1); of $\mathrm{g}(\mathrm{z})$ is a solution, its reflection about the y-axis, 1.e., $y(-z)$, is also a solution. In particular, if $y^{\prime}(0)=0, y(-z)=y(z)$, and if $y(0)=0, y(-z)=-y(z) ; 1.0$, , the re exist solutions even and odd about $z=0$. Hote that the possible use of sine for cosine in (1), 1.0. , a shift of the origin by $\pi / 4$, would have obscured this property: the reflection would st1ll be possible, but about $\pi / 4$ rather than $0,1 . \theta_{0}$, we would in effect have reverted to (1). Noto also that this property must be dropped if $\mathrm{J}(\mathrm{z})$ in the H1ll's equation is not even about any $\mathbf{z}$. 
Just as translations by $\pi$ can be extended to $2 \pi, 3 \pi$, etc., the reflections may be made about any $z$ about which the coefficient of ( 1 ) is even; i.e., about $\mathrm{z}=0, \pi / 2, \pi, 3 \pi / 2$, etc. Similarly, there exist solutions even and odd about these values of $\mathbf{z}$. A reflection about $\pi / 2$ is equivalent to combining a translation by $\pi$ with a reflection; $1 . e .$, $y_{2}(z-\pi / 2)=y_{1}(\pi / 2-z)$ is equivalent to $y_{2}(z)=y_{1}(\pi-z)$ o of course, a solution even about $z=0$ might be odd about $z=\pi / 2$, and vice versa.

A combination of $\mathrm{y}(\mathrm{z})$ with its reflection and/or translation might constitute a complete solution. However, more powerful properties are needed if, given a numerical solution in a finite interval, we are to extend it indefinitely. Thus, $y(z)$ and $y(z+\pi)$ are not linearly independent if $y(z)$ has a period of $\pi ; y(z)$ and $y(-z)$ are not linearly independent if $\mathrm{y}(\mathrm{z})$ is either even or odd; the solution might be nonperiodic; or it might have an uncomfortably long period.

\section{NORMAL (FLOQUET) MODES}

It was long perceived by the astronomers, and it was confirmed in 1883 by Floquet, that the re exists a solution of the form

$$
y(z)=\theta^{\mu z} \phi(z)
$$

where $\mu$ is constant and $\varnothing(z)$ is periodic, with the same period $(\pi)$ as that of the coefficient in (1). Such a solution can be readily extended indefinitely; and its reflection usually completes the solution.

A proof of this important relation would be desired even by the occasional student of Mathieu theory. However $r_{\&}$ it would avail him little to verify (2) by substituting it in (1): this would only lead a differential equation in $\phi_{3}$, which is burdened by the presence of the unlinown $\mu$ and is, in fact, the eigenvalue equation for $\mu$ (or for a); the periodicity of $\varnothing$ would still be far from obvious. The fact is in spite of the elegance of (2), - that $\mu$ and $\phi$ are not as basic concepts as the number $e^{14 \pi}$. The proof proceeds as follows.

Consider any two independent solutions, say $g(z)$ and $h(z)$, and let us try to construct a normal mode out of these two; $i_{0} \theta_{0}$, let it be

$$
\mathrm{J}(\mathrm{z})=\mathrm{Ag}(\mathrm{z})+\mathrm{Bh}(\mathrm{z})_{2}
$$

where, normalization aside, only the ratio of the constants, $A / B$, is of interest. Evaluate (3) at $z+\pi$. Observe that by the first fundamental property of solutions $y(z+\pi), g(z+\pi)$ and $h(z+\pi)$ all are solutions, and hence can be decomposed in terms of $g(z)$ and $h(z)$. In particular, put

$$
g(z+\pi)=g_{1} g(z)+g_{2} h(z)_{2}^{3} h(z+\pi)=h_{1} g(z)+h_{2} h(z)
$$


whe the coefficients $g_{1}, g_{2}, h_{1}$ and $h_{2}$ may be readily detemined, by differentiating $(4)$ and putting $z=0$. Then

$$
y(z+\pi)=\left(A g_{1}+B h_{1}\right) g(z)+\left(A g_{2}+B h_{2}\right) h(z) .
$$

On the other hand, if $y(z)$ is of the form (2), we must have

$$
7(z+\pi)=\operatorname{Sy}(z)
$$

where $S=e^{\mu \pi}$. Now, (5) and (3) amount to (6) only if

$$
\begin{aligned}
& \mathrm{Ag}_{1}+\mathrm{Bh}_{1}=\mathrm{AS} \\
& \mathrm{Ag}_{2}+\mathrm{Bh}_{2}=\mathrm{BS},
\end{aligned}
$$

which, however, can be solved for the ratio

$$
A / B=h_{1} /\left(S-g_{1}\right)=\left(S-h_{2}\right) / g_{2}
$$

only if

$$
\left|\begin{array}{cc}
g_{1}-s & h_{1} \\
g_{2} & h_{2}-s
\end{array}\right|=s^{2}-\left(g_{1}+h_{2}\right) s+\left(g_{1} h_{2}-g_{2} h_{1}\right)=0 .
$$

It remains only to solve (9), to define $\mu$ as $(\mathrm{lnS}) / \pi$, to introduce $\phi(z)$ as $e^{-\mu \pi} y(z)$ o where, of course, $y(z)$ is given by (3) and (8) - and to perform the easy demonstration thet $\phi(z+\pi)=\varnothing(z)$. The statement then is: given any $\mathrm{g}(\mathrm{z})$ and $\mathrm{h}(\mathrm{z})$, it is always possible to determino $\mu$ and $\phi(z)$ so that (2) will be solution. The inva riance of $\mu$ and $\phi(z)$ with respect to the choice of $g(z)$ and $h(z)$ may, for our present purposes, be accepted as obvious.

The reader will note that the Floquet theory is a generalization of the translatability of solutions, and that (6) is a simpler statement of this theory than (2). The statement may very well be: given any $g(z)$ and $h(z)$, it is always possible to determine $S$ and the ratio $A / B$, and to construct $y(z)$ such that $(6)$ will hold. Note that the reflectability of the solutions has not been used. 
Obviously, if the two values of $S$ from (9) are distinct, they determine the two normal modes, say,

$$
y_{1}(z)=e^{\mu_{1} z} \phi_{1}(z) \text { and } y_{2}(z)=e^{\mu_{2} z} \phi_{2}(z),
$$

the superposition of which completes the solution. This would also complete the theory in the very general case of a Hill's equation with a non-even $J(z)$. Note that, since the translatability of the solutions has been used up, $y_{2}(z)$ cannot be got by a translation of $y_{1}(z)$.

Considerable simplification is now afforded by the evenness of the coefficient of ( 1 ): viz., a second solution can be also obtained as a reflection of the first. In that case $\mu_{2}=-H_{1}$ and $\phi_{2}(z)=\phi_{1}(-z)$. The first condition means that the two values of $S$ are reciprocal, or that the last term in (9) must be $1:$ this indeed can be shown to be the case for any choice of $\mathrm{g}(\mathrm{z})$ and $\mathrm{h}(\mathrm{z})$, but in practice this ensues even more naturally - viz, it becomes apparent as soon as a practicable choice of $g(z)$ and $h(z)$ is made. The second condition, again, can be proved; but in practice it follows naturally when the second solution is taken to be a reflection of the first o which assumption, indeed, is in agreement with the first condition.

A case in which the reflection of a normal mode fails to furnish a linearly independent solution is a peculiar orphan of the boundary value part of the Mathieu theory, and will be discussed separately.

\section{PRACTICAL COMPUTATIONS}

Whittaker and watson ${ }^{6}$, whose proof of the Floquet theory is paraphrased above, make no commitment about the choice of the auxiliary functions $g(z)$ and $h(z)$. McLachlan pointed out ${ }^{*}$ the convenience of using the even and odd functions, say $\underline{\underline{Z}}$ and $\underline{\nabla}_{9}$ with what may be called unitary conditions: $u=I_{2} u^{p}=0_{j} v=0_{9} v^{0}=\bar{I}_{j}$ in which case $g_{1}=u(\pi)$ is the same as $h_{2}=v^{\theta}(\pi)$, and $(9)$ becomes

$$
S^{2}-25 S+1=0, \quad S=E \pm \sqrt{E^{2}-1}
$$

where $E \approx u(\pi) \approx v^{0}(\pi)_{9}$ but he discussed the theoretical calculations of $S$ much as though the numerical computation of both $\underline{u}$ and $\mathbf{v}$ had indeed to be carried over the interval $0<z<\pi$. In computing practice, of course, such work can be halved in a number of ways. Thus, in the available calculations $3,4,5$ the symmetry of $\underline{\underline{u}}$ and $\underline{v}$ is utilized to limit the

Reference $1_{2}$ pp. $23=24$. 
calculations to the interval $0<\mathrm{z}<\pi / 2$, the remainder being furnished by the reflections about $\mathrm{z}=0$; the interval then is $-\pi / 2<\mathrm{z}<\pi / 2$, and the unitary initial conditions occur in the middle, rather than at the beginning of the interval. Then ( 9 ) assumes the form

$$
S^{2}-2(2 A-I) S+1=0, S=2 A-I+2 \sqrt{A(A-1)},
$$

whe re $A=u(\pi / 2) v^{i}(\pi / 2)$. Incidentally, a running check may then be kept: that the Wronskian, $u v^{0}-u^{0} \mathrm{v}$, remains $i_{\text {; }}$ this concerns the very important (but now it is hoped, largely historical) question of the reliability of the high-speed computing machines. Note that the two values of $S$ are always reciprocal.

In retrospect it appears that if and when additional computations are to be made, at least three alternative procedures ought to be considered. Two of these, much alike, are obvious: may utilize, instead of the symetry, the fact that $u(\pi)=v^{\prime}(\pi)$, i.e., compute ei ther only $u(z)$ or only $v(z)$, but for the full period $0 \angle z<\pi$. This will forego the check on the Wronskian, but might save time in the subsequent handling of the machine data and in ressetting the machine for the second set of initial conditions, or some capacity of the machine. The second auxiliary function, when needed, can be gotten by the reflection of the first about $\pi / 2 ; e . g .$, as $U(z-\pi / 2)$. $\mathrm{u}(\pi / 2-\mathrm{z})$, which is the same as reflection about $\mathrm{z}=0$ and translation.

The third possible procedure is mentioned, as a matter of curiosity, for the reason that its details have somewhat better physical significance in relation to certain other problems covered by the Mathieu theory: if $u+$ iv $z \mathrm{re}^{i \theta}$, only $r(\mathrm{z})$ need be computed by integrating a differential equation, $\theta(z)$ being easy to compute by quadrature.

Arailable computations are limited to the equations ( $I$ ), but the methods discussed ${ }^{3}$, of course, are applicable to any Hill's equation, as we.11. It is hoped that they will also prove useful in extending the numerical. work to the other Mathieu equation (with cosh instead of cos), which belongs to the theory of functions of complex variable.

\section{CLASSIFICATION OF SOLUTIONS}

Three principal cases should be distingutshed accordingly as $\mathrm{E}^{2}$ is greater than, equal to, or less than 1 .

(a) Unstable Solutions. $E^{2}>1$.

This should be subdivided into

(I) the simpler case, $E>1$, both $S>0$ 。 The physical significance is that $\underline{u}$ (or $v$ ) either grows more or less monotonically, or, if oscillatory, has completed (or is

* The instability "in the large" i.e.g the fact that amplitude of $\bar{g}$ is unbounded, should not be confused with what may be called "local instability", which will be discussed presently. 
about to complete) at least one (or 2,3 , etc。) period; and that it is oscillating further than at the start. In fact, the essence of the phenomenon is resonance. $\mu$ is real (and is always reckoned as positive, i.e., is taken for the growing mode). One normal mode grows, and the other shrinks, exponentially. It can be established empirically (and confirmed theoreticaliy, with some effort) that the combinations of the parameters $a$ and $g$ at wich this situation occurs constitute a region, on the $a-q$ chart, which converges (for $q \rightarrow 0$ ) to the point $a n^{2}, n$ even integer. These may be called "evenoorder regions of instability". A special such region is the zeromorder region, or the region of "static" instability, characterized principally by a ne gative coefficient a: incidentally, a $\angle 0$ does not assure "static" instability: with certain values of $q$ oscillations may be stable even with negative a (cf. Fig. 2):

(2) The case of $\mathrm{E} \angle-1$ is not much more complicated. The physical significance is that u has completed approximately a hal fo integral number of periods, and the oscillations are growing; after the next interval $\pi$ of $z$, the resonance will be conspicuous again. Both values of $S$ are negative; but now, in order to account for a negative $s: e^{\mu \pi}, \mu$ must have an imaginary part of 1 (or gene ra $21 y_{2}$ an odd integer). The

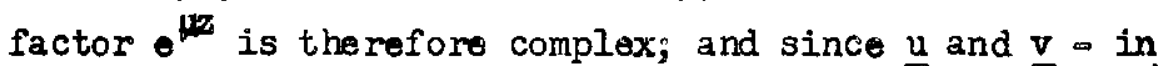
these computations, at least are real, the function $\phi(z)$ must be complex. It is conventional in this case to consider only the real part of $\mu_{2}$ and to combine the factor $0^{i\left(I_{\mu}\right) z}$ (where $I_{\mu}$ is the imaginary part of $\mu$ ) o which factor has a period of $2 \pi$ o with the complex $\phi$ of period $\pi$ into 2 real function $\phi(z)$ of period $2 \pi$. The regions in which this occurs converge to $a=n^{2}, n$ an odd integer, and may be called "odd-order regions of instability". The most important region of Mathiev-type instability (the firstoorder one) belongs to this class.

An inspection of the mechanism of the growth or shrinkage of the oscillations, as well as of the phase-relations involved, yields a considerabie insight into the physical significance of Mathieu theory. This will be attempted presently. The essence of the phenomenon is the tendency to lock into a resonance at an angular frequency $l_{9} 2,3, \ldots \ldots$ (i.e., a multiple of one half of the frequency of cos $2 z$ ), rather than at what may be callod "expected natural" angular frequency $\mathrm{a} / \mathrm{l} / 2$.

If the factor of 2 under the cosine symbol in ( 1 ) is omitted ${ }^{4}$, the numbers associated with the instability regions (i.e, their angular frequencies) become $0,1 / 2, I_{2} 3 / 2, \ldots \ldots$; $i_{\text {.e. }}$, one might then speak, 'rather ${ }_{9}$ of "half-integral" and "integral" orders of instability. 
Both cases can be merged ${ }^{6}$ if Floquet theory is applied to the interval $2 \pi$ (rather than $\pi$ ) of $z$; although that procedure fails to bring out the periodicity of $\phi(z)$ in $f u l l$.

(b) Stable solutions. $E^{2}<I_{0}$ ' While the magnitude of $u(\pi)$, or of $\nabla^{\prime}(\pi)_{2}$ is less than at the start, the physical significance of that is not 2 damping of the oscillations, but rather a change of phase; in fact ${ }_{2}$ the essence of the phenomenon is the absence of the resonance. Mathematically, the solutions are "bounded": although in certain regions of $z$ the beats can produce an impression of "local" instability, and might lead to very large (viz.g perhaps unexpected) values of $\underline{u}$ or $v_{0} I_{0} e_{.}$, while the boundedness assures that a regime of local, or apparent, instability will always be followed by a regime of local damping, the practical significance (presumably, especially in a close proximity to the instability regions) might be akin to instability。

The two values of $S$ are complex conjugates: but they are still reciprocal, and hence the magaitude of $S$ is $1: 1 . \theta . g$ the real part of $\mu$ is $0_{0}$. It is conventional, to designate such an imaginary $\mu$ by $1 \beta$, $\beta$ real. The meaning of $S=e^{i \beta \pi}$ is a rotation in the complex plane through angle $\beta \pi$. The factor $e^{i \beta z}$ is complex; and even if the normal modes were real (which, it will be presently shown, cannot be the case), $\phi(z)$ must be complex*. The value of $\beta$ as determined from $S$, of course, may contain an arbitrary even integer; and since there are two opposite values of $\beta_{9}$ $\beta$ is naturally confined to the range $0<\beta<1$. In fact, $a$ value of $\beta=1$ means simply a reversal of phase of $y(\pi)$ as compared with $y(0)$ : this means a change in the number of periods of $y(z)$ per period of $z$ by $a$ half-integer, or a change of the angular frequency of 1 : which is precisely what happens if we move across the stability region on the a.c plane from one instability region to the adjoining one.

If $\beta$ is a rational fraction $p / s$ in its lowest tems, after and only 2 fter a the increment $28 \pi$ of $z$ the vector $e^{i \beta z}$ will return to its starting position: $i_{0} e_{0}, \quad J(z)$ is theoretically periodic, but its period may be extremely large. If $\beta$ is irrational, $\mathrm{J}(\mathrm{z})$ is, strictly, non-periodic。 However, it would seem that if a very long (complex) helix of $\mathrm{y}(\mathrm{z})$ were available, one could detect in it a number of approximate periodicities, as an irrational $\beta$ may be approximated by a. sequence of tional numbers.

(c) Characteristic Conditions $B^{2}=I_{0}$ This important borderIine case is a rather exclusive domain of the boundaryovalue part of Mathieu theory。 Nevertheless $s_{9}$ two classes of solutions must be distinguished.

* Substitution in (1) shows $\phi^{\prime} \equiv 0$ if $\emptyset^{\prime}$ is $\mathrm{real}_{2}$ which is impossible. 
(I) Characteristic Solutions (the Mathieu functions), which are periodic. It is these solutions which have been the more extensively studied; in fact, the whole boundaryo value-problems part of Mathieu theory amounts to a search for solutions of this specified (periodic) character.

The physical significance of suct solutions suggests itself readily: if $u(\pi)$ or $v^{0}(\pi)$ is $\pm l_{g}$ the initial conditions for the interval $\pi<z<2 \pi$ might be practically identical. with the conditions at $z=0 ;$ in fact f $_{g}$ the second necessary condition, that $u^{B}(\pi)$ or $v(\pi)$ is $O_{2}$ is either supplied by the machine automatically (in which case the function computed is the characteristic solution) or follows from $u(\pi)=\nabla^{q}(\pi)$ and $u v^{0}=u^{q} \nabla=1$ (in which case the characteristic solution could be made out of the computed function and its reflection $\mathrm{U}$ or $\mathrm{V}$ ). In particular, in (ㄱ) , $S=+1$ implies 6 ither $A \equiv 1$ or $A=0$ : and considering the Wronskian, there are four o and only four - ways in which this may occurs at $\mathrm{z} \cdot \pi / 2$, either one of $u_{9} u^{\|}, v$ or $\nabla^{0}$ must be $O_{\text {. }}$ To establish the periodicity of the function in question it then remains only to refle ct it (evenly or oddly $y_{2}$ as the case may be) about $\pi / 2$. Thus

\begin{tabular}{|c|c|c|c|c|c|}
\hline $\begin{array}{l}\text { If } 2 t \\
z=\pi / 2\end{array}$ & $S=$ & $\begin{array}{c}\text { the characteristic } \\
\text { function is }\end{array}$ & $\begin{array}{l}\text { its period } \\
\text { is }\end{array}$ & $\begin{array}{l}\text { its angula } x \\
\text { frequency is }\end{array}$ & $\begin{array}{l}\text { and it is } \\
\text { denoted by }\end{array}$ \\
\hline $\mathfrak{u}=0$ & -1 & u & $2 \pi$ & 1 & $\infty_{2 m+1}(z, q)$ \\
\hline$u^{\prime}=0$ & 1 & $\mathbf{u}$ & $\pi$ & 2 & $\operatorname{cec}_{2 m}(2, q)$ \\
\hline$v=$ & 1 & $\nabla$ & $\pi$ & 2 & $s e_{2 m}(z, q)$ \\
\hline$v^{\prime}=$ & -1 & $\nabla$ & $2 \pi$ & 1 & $\operatorname{se}_{2 \pi, \pi}(z, q)$ \\
\hline
\end{tabular}

These are the Mathieu functions of integral order. The notation $\mathrm{Ce}_{2}$ se is read "elliptic cosine" and "elliptic sine". The subscript, or the prder of the function, is the order of the instability region bordered by the charecteristic condition in question. While these functions have $\pi$ or $2 \pi$ as their exact period, much more prominent (and from the view point of physical significance, more important) is the osciliation of the frequency $n_{\text {, viz. }}$ of the order of the instability region. Thus in ${ }^{c e}{ }_{0}(z ; q)$ a conspicuous feature (in addition to an oscillation of period $\pi$ ) is the constant term: and $\operatorname{ce}_{n}\left(z_{8} q\right)$ rather resembles $\cos (\mathrm{nz})$ in its appearance, while $\mathrm{se}_{n}\left(z_{g} q\right)$ resembles $\sin (\mathrm{nz})$. The fundamental frequency is manifested only as beats, which - like the constant term of ${ }^{c \theta_{2 m}}$, in of 0 o are often barely discervible. At $q=0, c \theta_{n}$ and $s \theta_{n}$ indeed degener te (normalization aside) to $\cos (n z)$ and $\sin (n z)$. 
With the trigonometric and the hyperboife functions of similax names, ce and se share only the basic properties of evenzess and oddness, and of a mutual orthogonality. They do not have the convenient properties of $\sin _{2} \cos$, sinh and cosh as far as their derivatives, or the relations between their sajares, are concerned.

It is important to note that $c \theta_{n}$ and $s \theta_{n}$ ares not the solutions of adenticaliy the same equation. Each instability region of ordeis $n_{9}$ is $f O_{2}$ is bounded - for the samo $q$ - by two characteristic conditions, wits differen values of a of (1) and each characteristide function belongs to one or other of these two conditions. Specifically, if $\mathrm{g}$ is reckoned as positive (as is conventional), ce belongs to the "far" side of tho instability region (the greatex value of a), and se belongs to ths "near" side (the lower a). The situation would be reversed if $\mathrm{g}$ were reckoned as negative, Viz., if (1) were written as $y^{-1}+(a+2 q \cos 2 z) y=0$; but the shapes of the timo curves would then be exchanged: viz, $s_{1}$ would then look like the ce. solution of (I), and vice versa, except for the change of phase: $i_{\circ} e_{2}$, the change would amount to shifting the origin of $\mathrm{z}$ by $\pi / 2$.

In fact, it is much easiex to consider the physical significance of these two solutions. Thus, the odd se is the more eccnomical of the "static" stabilityr, as represented by $a_{0}$ when the spring stifiness is a mirimum $s e_{1}$ passes ' tinrough zero, $i_{0} \sigma_{0}$, is in a phase when the spring stiffness does not matter anyway: and $s_{1}$ utilizes the maximm of the spring atirfness in full, $i_{0} \theta_{0}$ the maximum of $s \theta_{2}$ occurs ' when the spring stifiness is a maximum. In contrast, the eren se ${ }_{1}$ is the more wasteful of the static stallity, or is the more sluggisin function: to wito it fails to utilizo the greater value of a to inerease its frequency: it passes through zero when the spring stiffness is a maximum, and the refore dwelis longez for a more exactily a dwell.s jugt as long, in spite of the greater value of \&) at eash swing from 2ero. Thus, as we proceed across an instabilitiy region in the direction of increasing $a$, the re is ro change in erequency, but there is a change in phase, as the excess of static siability is being used less effectively.

This explanation can be extended to higher orders of the instability regions: but there the split in the static stability values matters progressively less and less. 
The zero-order region is rather an exception. It is bounded only on the "far" side, and has only the even solution ce, of period $\pi$ (i.e., of twice the frequency of $\left.\mathrm{ce}_{1}\right)$; there is no $s \theta_{0}(\mathrm{z}, q)$. A change of $+q$ to $-q$ mere $y$ shifts the origin of $z$ from the minimum to the maximum of $c \theta_{0}(z, q)$.

The characteristic solutions being either even or odd, their reflections fail to furnish linearly independent solutions. This is not in any contradiction to the Floquet theory, since the existence of both auxiliary functions $g$ and $h$ are by no means essential to that theory. Thus, if (2) Is to be applied to $g(z)$, only, and $S$ is to be $I_{g}$ there merely follows that $B=0$.

The characteristic solutions definitely belong to (and in a sense, are ancestors of) the stability regions. Specificaliy, if wo restrict the period to $\pi(S=1)$, the re arise only the border lines of the even regions of instability; if we relax this to 211 ow $2 \pi \quad(S=-1)$, the re arise the border lines of odd regions of instability: if to $4 \pi \quad(S=i)$, the lines of $\beta=1 / 2$ if to $3 \pi \quad\left(S=e^{12 \pi / 3}\right)$, the lines of $\beta=2 / 3$, etc., until all stability regions are densely shaded. The even and odd stable solutions are, in fact, called Mathieu functions of fractional order $n+\beta$. The discontinuity of this shading due to the possibility of an irrational $\beta$ would appear, to a "technologist"*, a rather technical matter.

With the unstable solutions the characteristic solutions share, of course, their predominant frequency; but they are distinguished by their very definite phase relations, viz., their evenness or oddness. In fact, the even and odd unstable solutions (our $u$ and $v$ ) are denoted by ceu and seu (u for "unstable"), with $a n$ index $n+\mu$; where the two conditions (two values of a for the same $\mu$ and $q$ ) can be distinguished ${ }^{* *}$ by writing $\mu$ or ${ }^{*}$.

Curiously, the uniqueness of the characteristic solutions (viz., the absence, for a given $a$ and $q_{\text {g }}$ of a second periodic solution of the same frequency) seems to have been proven, by Ince, only as late as 1922 .

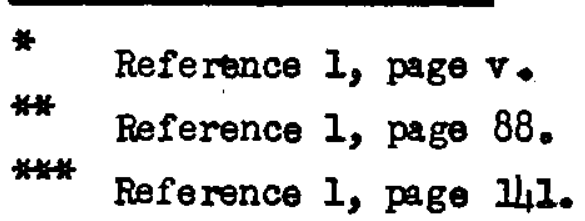


(2) Secular Solutions. With characteristic conditions, as we have seen, the characteristic solutions require particular (either even or odd) initial conditions. But from the initialovalue point of view the non-characteristic initial conditions are just as legitimate: in fact, these "second non-periodic" solutions, to which such conditions give rise, have been given a standard notation: when $u$ is $\mathrm{ce}_{n}\left(z_{9} q\right)$, the odd solution $\underline{v}$ is denoted by $f e_{n}(z, \bar{q})$, and when $v s e_{n}(z, q)$, the even $u$ is denoted by $g e_{n}(z, q)$; the English names are apparently lacking. These solutions are oxphans in the sense that they arise only at the characteristic conditions, get fail to satisfy the basic requirement of the boundary value theory, which is the reason for these conditions: they are neither periodic, nor stable, nor do they obey the Floquet relation (2). On the other hand, they do obey an analogous relation, which apparentily should be called the Ince relation:

$$
\begin{aligned}
& f e_{n}(z, q)=C_{n}(q) z c e_{n}(z, q)+f_{n}(z, q) \\
& g e_{n}(z, q)=s_{n}(q) z s e_{n}(z, q)+g_{n}(z, q)
\end{aligned}
$$

where $C_{n}$ and $S_{n}$ are, for a given $g_{g}$ constant coefficients, and the functions $f_{n}$ and $g_{n}$ are periodics of the same period as the corresponding characteristic functions, and are, respectively, odd and even. Just as (2) has the more basic form (6), the more basic form of (12) states that the increment of $f e_{n}(z, q)$ or ge $(z, q)$ over any period $\pi$ (or $2 \pi$ ) is a constant, iot., the increment is a periodic function of 2. This relation being of a lesser generality than the Floquet's one, its proof is here omitted; it may only be remarked that the proof utilizes reflectability as well as the translatability (since the reflectability is already inherent in the fact that $\mathrm{ce}_{n}$ and $s e_{n}$ are symetric). These solutions are obyiously analogous to the secular solutions (such as $x e^{x}$ ) of linear differential equations with constant coefficients.

The lack of periodicity of $\mathrm{fe}_{\mathrm{n}}$ and $g \mathrm{e}_{\mathrm{n}}$ is apparent: for small $z$ they have the phase of $f_{n}$ and $g_{n} i_{0} e_{0,}$ are $\|_{2} t 90^{\circ}$ to $\mathrm{ce}_{\mathrm{n}}$ or $\mathrm{se}_{\mathrm{n}}$ as the case may be: while for la rge $\mathrm{z}$ they approach the phase of $\mathrm{ce}_{n}$ or $s e_{n}$ asymptotically. The lack of stability, as $\underline{z}$ approaches $\pm \infty 2$ is made obvious by the

Reference $1, \mathrm{pp} 4$ and 14 . 
presence of the factor $\underline{z}$. Since the ratio of any two successive maxima of $\mathrm{fe}_{\mathrm{n}}^{-}$and $g e_{n}$ approaches $l_{9}$ for all practical purposes, these solutions tend to $\mathrm{ce}_{\mathrm{n}}$ and $\mathrm{se} \mathrm{n}_{\mathrm{n}}$ of some large amplitude.

The secular solutions seem to constitute the border line between the slightly unstable solutions and those "stable" solutions which lead to large amplitude of $\mathrm{g}(\mathrm{z})$ for large z. So to say, $2 s$ we pass across a characteristic condition from the stable region into the unstable one, the beats may generally grow in magnitude indefinitely, and at the same time recede indefinitely: within a large finite interval of $\mathrm{z}$ the solution may finally become indistinguishable from a secular one: thereafter it will pass into an exponentially unstable solution. If, how ever, the phase of the initial conditions is exactly right, the beats may be moderate in magnitude, will pass through the characteristic solution, and then shrink exponentialiy.

Obviously, fe ${ }_{n}$ and $g e_{n}$ can be extended indefinitely if the coefficients $C_{n}$ and $S_{n}$ and the functions $f_{n}$ and $g_{n}$ are determined in a finite interval: specifically, if the calculations are extended to a point at which either one of $f, f^{\prime}, g$ or $g^{\prime}$ is zero.

Of course, the occurrence of the characteristic conditions in the numerical calculations is either in the nature of an accident, or must be imposed specifically. While some of the available calculations pass quite close to a characteristic condition, none of them do so exactly; for such an $a b$ ovo derivation, check and inspection of the characteristic conditions has not been the purpose. It is nevertheless conceivable that extensive data on $\mathrm{Ce}_{n^{2}} \mathrm{se}_{n}$, $f_{n}, g_{n}, C_{n}$ and $s_{n}$ can be procured. Incidenta $11 y_{9}$ the functions in question seem to be tailor made for an "empirical" analysis in Fourier series, so that each one of these functions of $\mathrm{z}$ may be given in a compact column of Fourier coefficients. The method will be free from the difficulties of convergence with la rge values of $q$.

It is interesting to note an empirical observation made in the construction of the contour plots of $S$ in the instability regions: in vicinity of the characteristic conditions $25 / 2 a$ and $2 S / \partial q$ appear to be infinite. If proved, this will mean that an accurate determination of the chacteristic conditions might be aided by the sensitivity of $s$ to the choice of $\underline{a}$ and $q$. 
Similarly, no information is now available on the curious question, whether the re exist some convenient relations between the periodic functions ${ }^{c e_{n}}{ }_{n} s e_{n^{2}} f_{n} g_{n}$ and their derivatives. The intexpretation of the solutions in terms of infinite series is extremely laborious, and the empirical numerical apprach might, be of a considerable val us.

\section{PERIODIC KERNELS}

The outlined and the available machine computations have been congerned mainly with the determination of the Floquet coefficient $3 \mu_{\mu}$ of $(2)$, or ${ }^{5}$ of the "amplification factor" $S$ of (6). The solutions which can be extended indefinitely in this manner, of course, are only the normal (Floquet)

modes*; but the readily obtainable auxiliary functions, viz., the even

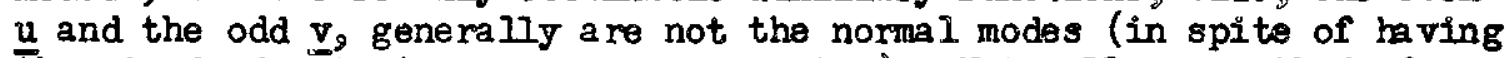
the standard notation $c_{n+\beta}, \operatorname{cen}_{n+\mu}$, et.c.). Naturally, a method of extending the solutions, to be complete, should include a specification of either a mormal mode, or a preferably a of the function $\phi(z)$ of (2).

To specify a normal mode, it remains merely to carry out the operations (8) and (3). If one nomal mode is $A u+B v_{9}$ the other one is $\mathrm{Au}=\mathrm{Bv}_{2}$ both with a possible arbitrery (complex) factor.

In particular, with the unitary conditions on one end of the $\pi$ interval, (8) becomes

$$
A / B=v(\pi) /(S-u(\pi))
$$

which may be transformed, with the aid of (10) and the Wronskian, into forms invalving either only $\underline{u}$ or only

$$
A / B=E^{2} / u^{B}(\pi) \sqrt{E^{2}-1}=v(\pi) / \sqrt{E^{2}-1}
$$

With the unitary conditions in the middle of interval, as in (II), (8) becomes

$$
A / B=v(\pi / 2)(S+1) / u(\pi / 2)(S-1)
$$

At a characteristic condition a normal mode is either Au or Bvg in fact, that $A / B$ is infinite when $u$ is $c e_{n}$ is obvious from (1/i): that $A / B$ is zero when $D$ is ${ }^{n}$ can be seen from $(13)_{2}$ for then $u(z)$ is $g e_{n}(z)$ and is not periodic, and the refore $u(n) \& \mathbb{L}_{0}$

* Not to mention the secular (Ince) modes. 
The auxiliary functions $u$ and $v$ being real, in the unstable regions $A / B$ and the normal modes are real; it does not particularly help matters to introduce the complex spirals $u+i v$ or $y_{1}+i y_{2}$. It may be remarked that the normal modes, as well as $\phi(z)$, are then neither even nor odd changing as they do across the unstable region from an odd $s e_{n}$ to an even ${ }^{\infty} n^{*}$

In the stable regions a convenient visualization is possible. Since the vector $S$ has a magnitude of 1 , the vectors $S+I$ and $S-I$ in (15) aremtually perpendicular in the complex plane, and therefore the ratio $A / B$ is a pure imaginary; thus the normal modes cannot be real. The convenient feature is that the complex helices representing these normal modes have $u$ and $v$ as their rectangular components o except that one of these coordinates must be stretched in particular ratio (the magnitude of $A / B$ ), and that the whole helix may have an arbitrary scale and phase (that of either $A$ or $B$ ). In other words, if we put $A=I_{2} u$ can be considered as simply the real component of either one of the complex conjugate normal modes ${ }^{\circ} r_{9}$ if we put $B=i, V$ can be considered as simply the imaginary component of one of these modes (the other helix being a mirror image of the first). The points on the helix of a normal mode corresponding to $z_{2} z+\pi, z+2 \pi$, ete. indeed lie on uniform helices; but the whole helix, nevertheless, is not a uniform one - because of the oscillations of the magnitude, and the nonouniform rotation, of the vector $\phi(\mathbf{z})$.

The specification of $\phi(z)$ is preferable to a specification of a normal mode over a complete period, for two reasons. In the unstable regions $y(\pi) \neq \pm y(0)$, nor is $y^{B}(\pi)$ the same as $\pm y^{g}(0)$, so that if $y(z)$ were to be expanded in Fourier series*, it must be reskoned as discontinuous. In the unstable regions it is entirely impractical to tabulate $\mathrm{y}(\mathrm{z})$ over the complete period $(2 \mathrm{~s} \pi)_{2}$ while a reconstruction of $y(z)$ from the data in the interval of length $\pi$ amounts to the use of the function $\phi(z)$.

There exist a number of ingenious methods of determining $\phi(z)$. Thus, apparently ${ }^{*}$ in 1914 Whittaker developed a Fourier series in terms of a new parameter $\sigma$, whose physical significance is in the nature of a shift in phase; in terms of $\sigma$ and $q_{2}$ by the use of $a$

The function $\phi(z)$, obviously, is particularly suited for a Fourier series representation. While the purpose of this report has been to avoid the infinite series, the Fourier series, of course, hold an exceptional place among such - because of the strong physical significance of their coefficients.

Reference $I_{2}$ p. 70 . 
number of a willary series, it is possible to compute the coefficients $a$ and $\mu_{2}$ as well as the Fourier coefficients of $\phi(z)$. Melachlan gives what may be called Fourier Hathieu series, in terms of $\mathrm{ce}_{n^{2}} s \theta_{n}$. Particulariy useful, perhaps, are the recursion formulas for the Fourier coefficients of $\mathfrak{b}(\mathrm{z})$, which leave an a priorf computation of only four scalar Fourier coefficients necessary, the remainder being easily generated by the recursion.

Nevertheless it appears that for practical purposes the re remains room for the less imaginative (though heretofore more laborious) "empiriral" approach: viz. for a tabulation of numerical data, through computing as ( $\mathrm{Au}+\mathrm{Bv})_{0}^{-\mathrm{H}_{3}}$, and, if desired, decomposing this function in the Fourier series numerically. Such an underotaking (first outlined, apparently, by Brainerd) is outside the scope of this report: but the 1500 ENIAC trajectories $4_{25}$ are arailable to those who would ombarik upon it. In such an undertaking, the scalar components of the oscillatory Fourier integrand $\phi(\mathrm{z}) \mathrm{e}^{2 \mathrm{pz}}, \mathrm{p}=\mathrm{I}$ ana 2 , can be readily got from the availablo data at 31 points in the interval $\pi / 2$ of $z_{0}$ the higher-order coefficients ( $p \otimes 3,4 \% \ldots$ ) can be got by recursion. Incidentally, the interpolation (if desired) might be facilitated by reverting to polar coordinates: if $\phi \times \mathrm{re}^{i \theta}$, both the radial and the angular coordinates of the integrand, $r(z)$ and $\theta(z)+p z_{2}$ are smoother functions of $\mathrm{g}$ than its roctangular coordinates are.

A similar ampirical approach can be readily worked out for the Ince functions $f_{n}$ and $g_{n^{\circ}}$ However, since no available solutions occur exactly at the characteristic conditions, some interpolation across these conditions might be necessary: and in view of apparently peculiax behaviour of the solutions in this vicinity, the subject appears to deserve a separate study, as well as some additional numerical data.

As was mentioned before, the predominant frequency of $\phi(z)$ in the unstable regions (including the characteristio conditions) is that of the ordar of the region. It is natural to expect that in the stable regions the predominant frequency of $\phi(z)$ is that of the adjoining lowerourder unstable region, the factos $e^{i f 2}$ making up the differ ence between that frequency and the predominant (intermediate) freouency of the normal mode.

An interesting question is whether at some conditions the variation of $\varnothing$ may not amount to the change of phase only: offohand, this does not appear possible, since the shape of must also change from the economical (leptokurtic) $s e_{n}$ to masteful (platykurtis) $c_{n}$.

\footnotetext{
Refererios I, p. 65
} 


\section{REMAR RIS}

The Mathieu equation (or the Hill's equation with an even $J$ ) is particularly interesting because it constitutes a border-line between the linear and the non-linear differential equations. Specifically, at the characteristic conditions of the first-order instability region (and with a further stipulation that $2 q<a$ ) the "spring stiffness" coefficient of (I) is uniquely related to the magnitude of the characteristic function. The mechanism of the oscillation then is precisely the same as if this coefficient were a function of $\mathrm{se}_{1}$ or $c_{1}$; i.e., as though the equation were non-linear, $y^{\prime \prime}+y F(y)=0$. In particular, on the "near" side of the instability region, which gives rise to $\mathrm{se}_{1}$, the stiffness increases with the deflection (as in a tension spring), i.e., the curve of $\mathrm{yF}(\mathrm{y})$ is convex toward the axis of $y$; while ce, being less economical of the static stability, represents an osciltation such as might be caused by a compression spring ${ }^{*}$. The periodicity of $\mathrm{ce}_{1}$ and $\mathrm{se}_{1}$ is in agreement with the feeling that a system described by such a non-linear equation ( $F$ positive) is conservative and has no damping. The existence of two values of the static stability a for the same frequency confirms the feeling that the average value of $F(y)$ is not a good one for the estimate of frequency, or that the shape of $F(y)$ can affect the frequency. The usefulness of such an analogy, of course, is limited.

Particularly interesting is the mechanism of the shrinkage and growth of oscillations in this first-order region near exact resonance $(a=1)$. The shrinking mode then is receding from the equilibrium when the spring is stiffer $(\pi / 4<\mathrm{z}<2 \pi / 4)$, and is approaching the equilibrium $\mathrm{z}<\frac{3 \pi}{4}$ when the spring is weaker $(-\pi / 4<\mathrm{z}<\pi / 4)$. The result is a damping of the oscillation, somewhat similar to that produced by a damping force proportional to the velocity $\mathrm{y}^{p}(\mathrm{z})$ - with the important difference, that in the swing from one extremum to the next one, this effective damping force is applied to the shrinking Mathieu mode, so to say, in two spurts instead of one: viz., at $y(z)=0$ there is no force, while in the damped harmonic motion the damping force is maximum at that instant. The frequency of the oscillation then, generally, is not affected. The extremum occurs at $z=3 \pi / 4$.

The situation is exactly reversed for the growing mode: it recedes from equilibrium at the time when the spring is the weakest, and returns when the spring is the stiffest. The extremum occurs at $\mathbf{z}=\pi / 4$ o

The analog implied here is a familiar one: a point mass slides on a table and is connected by a spring passing through a hole in the table to a spring which is fixed on the other end. A familiar ballistic instance is the non-linearity of the righting torque on a fin-stabilized projectile. 
Again, there is little not effect on the frequency. Note thet both normal modes are neither even nor odd.

Consider then the situation when the extremum of the oscillation coincides with an extremu of the spring stiffness, $i_{0} e_{0}$, occurs at $2=0$ or $\pi / 2$. Thes there is no damping: we would say that both normal modes are present in equal proportions, and the shrinking of one is offsetting the growth of the other. However, the phase of the oscillation then is not stable, viz., this phase relation cannot be maintained. In particular, let the extremum of the osciliation occur at the maximun of the spring stiffness (at $\pi / 2$ ): the osvillation then is the nore sconomical of the static stability a, which in this case is greater than that corresponding to $\mathrm{se}_{1}$; therefore the phase of the oscillation will get ahead of the phase of the spring stiffness, or the extremum of $y(z)$ will shift toward $z=\pi / 4$. Now let the extremum occur at the minimum of spring stiffness (at $\mathrm{z}=0$ ): the oscillation then is analogous to co but lacks the margin of the static stability associated with the wastefin ${ }^{c e_{1}}$. The phase of the oscillation then will lag behind that of the spring stiffness, and the extremum of $y(2)$ will shift toward $z=\pi / 4$ again. This means that in both cases the growing mode will predominate. It can be said that the phase of the shrinking mode is in an unstable equilibrium while the phase of the growing mode is stable.

Next, it is interesting to inspect the mechanism of locking into resonance. Iet us consider, for instance, the case of static stability a being less than $l_{8}$ but still greater than that which gives rise to s $\theta_{1}$ (in the conventional notation, the case $a_{1}<a<1$ ). Let us inspect

the fous representative (but now no longer normal) modes which have their extreme at, $2=0, \pi / 4, \pi / 2$, and $3 \pi / 4$ (covering the period $\pi$ of $\cos 2 \pi)$. We may call these modes, respectively, "Wasteful", "Growing", "Economica]", and "Shrinking". It is natural to represent these phase relations as points of the compass (using, perhaps, the term "Nourishing" or "Nascent", eic, for "Growing" to complete the memonic symmetry). Obviously, the oscillation of the W-mode will lag (viz.g the vector representing its phase will move clccikwise on the diagram). On the other hand, the Emosciliation will advance (move counterclockwise). The So and $G$ (or N) -ogcillations will tend to have the "expected natural" frequency $e^{1 / 2}$ and will the refore lag. Thus there will eventually ensue in the $\mathrm{N}-\mathrm{E}$ quadrant a stable growing modes while in the $S=0$ quadrant the re may exist, with its phase in unstable equilibrium, a shrinking mode. For $a \rightarrow a_{1}$, both modes will tend to ${ }^{2}{ }_{1}$.

Similarly, if a $7 I$ but less than that which gives rise to $\mathrm{Ce}_{I}$ (denoted by $b_{1}$ ), the $\mathrm{N}$ - and $\mathrm{S}$ - modes will advance in phase, and the stable modes will exist in the $N-W$ and $S-W$ quadrants, tenoing to $W$-mode $\left(\mathrm{ce}_{1}\right)$ for $a \rightarrow b_{1}$. Since the stable modes are off the NoS phase, their rates of growth and shrinkage ( $\mu$ ) are naturally diminished. 
It will be noted that these non-normal nodes have a changing frequency; the beats between these frequencies produce other, "local", frequencies. In particular, it, is natural to expect that in a finite interval of a the apparent "loca?" frequency might be closer to that of al/2, rathere thas to the resonant erequercy, 1: this wolld be explained by the shift of phase resulting from the exchange of the magnitudes of the normal modes. The resonant

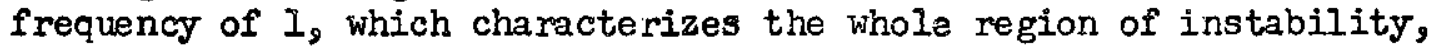
is manifested only as the frequency of the surviving mode.

\section{DESCRIPTION OF AVAIIABLE TRA JECTORIES}

While a generous amount of computation examples, tables, graphs and charts is given in McIachlan's book (including, particularly, Dr. J. Comrie's iso- $\beta$-pchart, or the contour plot of iso- $\beta$ and isom $\mu$ lines on the Ince's $a-q$ chart), it is here desired to describe, in particular, two sets of ENIAC data:

(1) Brainerd's data 4 . The equation (1) is written in the form

$$
a^{2} y / d t^{2}+\epsilon(1+k \cos t) y=0
$$

so that $t=2 z, k=-2 q / a$ (origin of $z$ shificeo by $\pi / 2$ ), $\in=a / 4$. The auxiliary functions $g$ and $h$ (our $u$ and $y$ ) were computed at intervals .0004 of $t_{\text {, to }} 10 \mathrm{places}$ (are recorded and a re apparentiy available, together with values of $g^{0}$ and $h^{1}$, at intervals of ol of $t_{9}$ probably to 7 places; cited to 5 places) for $\epsilon=1,2,3, \ldots \ldots 10^{-}$and $k=0.1$, $0.2, \ldots \ldots \ldots 1.0$. Also cited are values of $\mu$ (which in our notation is $\beta+i \mu$ ) and of $M$ (in our notation $B / i A$ ). While the mesh of $k^{\prime} s$ seems to be amply fine, the mesh of $E$ 's seems rather coarse; most points fall in the stablo regions. An $\sqrt{\epsilon}$ vs $k$ chart (i.es, the chart of $-2 q / a$ vs $a^{1 / 2} / 2$ ) is outlined. An extremely laconi.c summary of theory from the viewpoint of electrical engineer.

(2) BRL $d 2 t a^{5}$. The oquation (1) was used in the form

$$
d^{2} y / d t^{2}+(1 / 4)\left(x^{2}+\gamma \cos t\right)+0_{2}
$$

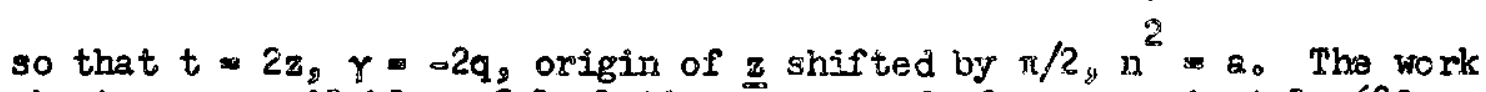
sheets are avaliable. Calculations were ma de fox approxima tely 680 combinations of $n^{2}>0$ and $\gamma$, as marked by cjecles on Figure 2. The work sheets state the two values of $S$ in the unstable regions, and the value of $A$ of (II) in the stable regions. 
The attaghed iso-S- $\beta$ chart is essentially the same as Comrie's iso $\beta-\mu$ chart 1 , with the following differences:

(1) The contour lines in the regions of instability are for the round values of $S=e^{\mu \pi}$, rather than for round values of $\mu$. As it was mentioned before, $S$ and (6) seem more basic than $\mu$ and (2); besides, $S$ is easier to compute than $\mu$, is al so much eavier to visualize, and probably - easier to use. Incidentally, it may appear thet in order to complete the symmetry, in the stable regions it might have been proper tc cite the vaiues of the angle $\beta \pi_{9}$ rather than of the coefficient $\beta$; however, in that case the factor $\pi$ is merely the statement of the most relevant unit (halfoturn instead of radian).

(2) The scale of abseissa is $a^{1 / 2}$ (rather than a), designated by ng this is Brainerd's $2 \epsilon^{1 / 2}$. This scale was suggested by McLachlan, but used for the first time, it appears, by Brainerd. The advantages of this scale are obvious: the number $n$ has a simple physical significance: the most important region of instability, the first-order one, can be magnified on the chart; the less important regions are shrunk: the scaie of $\beta$ along the axis of abscissas is uniform. However, there are also certain disadvantages: viz., the region of static instability, $2<I_{2}$ is perforce excluded.

(3) The ordinate is $r / n^{2}$, or $-2 q / a$, or Brainerd's $k$, ther than $\gamma$ or 2q. This again seems to have been suggested by McLachlan, who pointed out that in certain problems covered by Mathieu theory $q / a$ has a better physical significance than gs i.e., it is often desirable to inspect the line $q / a$ const rather than the line $q=$ const. In particular, with the change of the scale of abscissas from $a=n^{2}$ to $n$, the line $q / a=$ const becomes a parabola. The reader who prefers $g$ or $r$ as the parameter may easily construct, or use, the lines of $\gamma=\mathrm{kn}^{2}=$ const. The convenience of the scale of $k$ is that the regions of higher order of instability are shrunk, and the firstworder region can be magnified; thus, more information is brought within the rectangle of the chart. However, all intercepts of the characteristic lines with the line $n=0$ now recede to infinity, and these lines become asymptotic to the axis $n=0$. Thus for certain problems of Mathiau theory the iso-S- $\beta$ chart cannot replace the iso- $\beta-\mu$ chart.

It should be noted that Brainerd's and BRL's shift of the origin of $z$ by $\pi / 2$, i.e.g their use of a negative $g$ in (1), does not matter at all as far as this chart is concerned. However, as a me re matter of adhering to a standard notation, it should be noted thet at the characteristic conditions Brainerd's $\mathrm{g}$, for instance, does not approach ce ${ }_{n}\left(z_{9} q\right)$, as might be expected off-hand; rather, it approaches $s \theta_{n}(z-\pi / 2, q)$ when $n$ is odd, and $c_{n}(z-\pi / 2, q)$ when $n$ is even; similarly, his $\mathrm{h}$ approaches $-\infty_{n}(\mathrm{z}-\pi / 2, q)$ with $\underline{n}$ odd, and $-\theta_{n}(\mathrm{z}-\pi / 2, q)$ 
when $\underline{n}$ is even. The reader is further reminded that throughout this elementary review we a re not concerned with the normalization of the characteristic functions, while in the literature ${ }^{l}$ definite methods of standardized normalization 7 have been proposed.

In the study of the unstable regions a simple visualization of the chart is possible. The stable regions may be visualized at sea level $(S=1)$, and the unstable regions, as mountain promontories rising out of that sea. It is particularly interesting to inspect the domain $k<1$, viz., when the spring stiffness is always positive. In this doma in the first-order region, or the ridge, is readily seen to be most important, particularly at lower values of $k_{\text {; }}$ its crest, or tershed, is practically straight, while the higher-order regions terminate in sloping sand flats (and that in spite of the change of scale from $a$ to $k$; on the Mclachlan-Comrie chart this is even more so). On the other hand, for $k>l$ the higher-order regions (a domain which is way off the McIachlan-Comrie chart) apparently become more important than the firstmorder region. Also, as was mentioned above, at the edges the surface of these ridges seems to be vertical, even wi th the higher-order regions (as though the shores of even the sandflats were washed off).

Some visualization is also possible for the stable regions, although it becomes quite clumsy if we would attempt to blend it with the visualization of the unstable regions (this is natural, for $S$ in the unstable regions and the angle $\beta \pi$ in the stable regions ha ve different physical significance). It seems best to limit such visualization to the proximity of the shore line; then iso- $\beta$ lines are contours of the sea bottom. Again, it seems to be an empirical fact (possibly useful in a study of the vicinity of the characteristic conditions) that at the shore lines the surface of this bottom is vertical. In such a study, then, it might be advantageous to revert from iso-s lines to iso- $\mu$ lines.

The accuracy of this chart is severely limited by the crude graphical interpolations used, and does not do justice to the sevenplace ENIAC data.

The writer is indebted to Mrs. Florence B. Goggins for the construction of the charts.

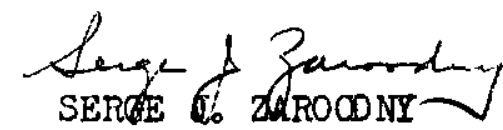





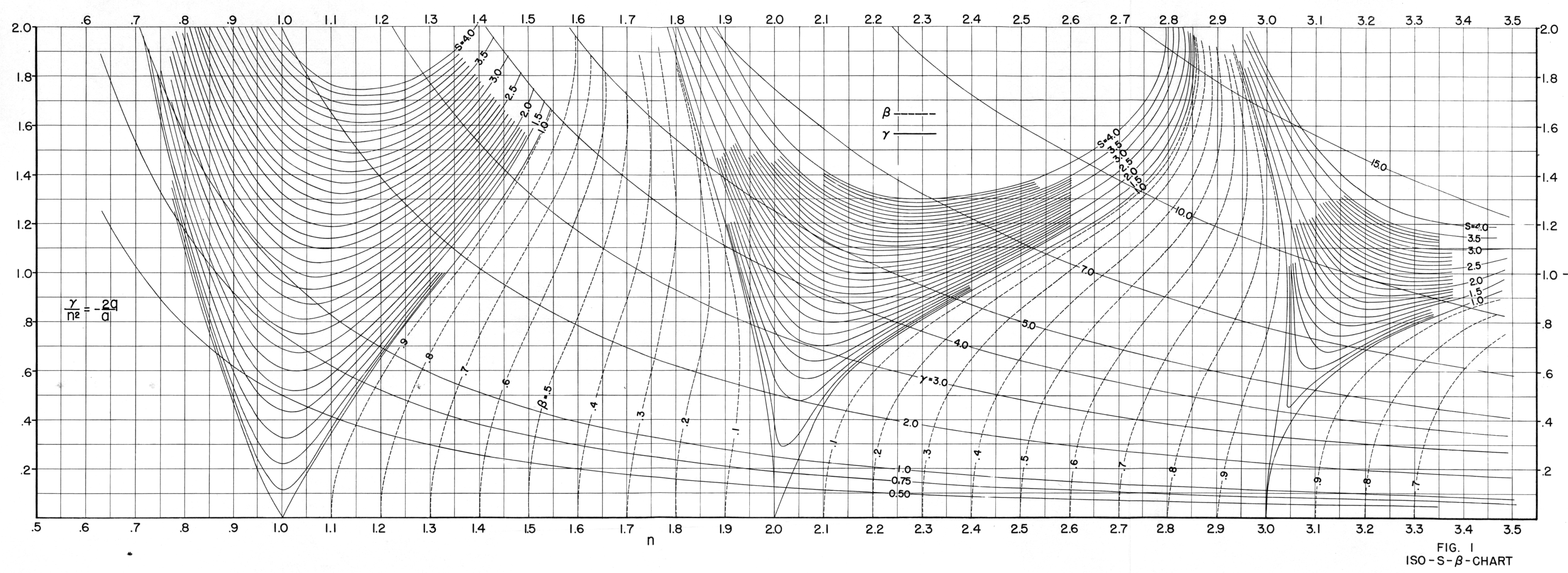




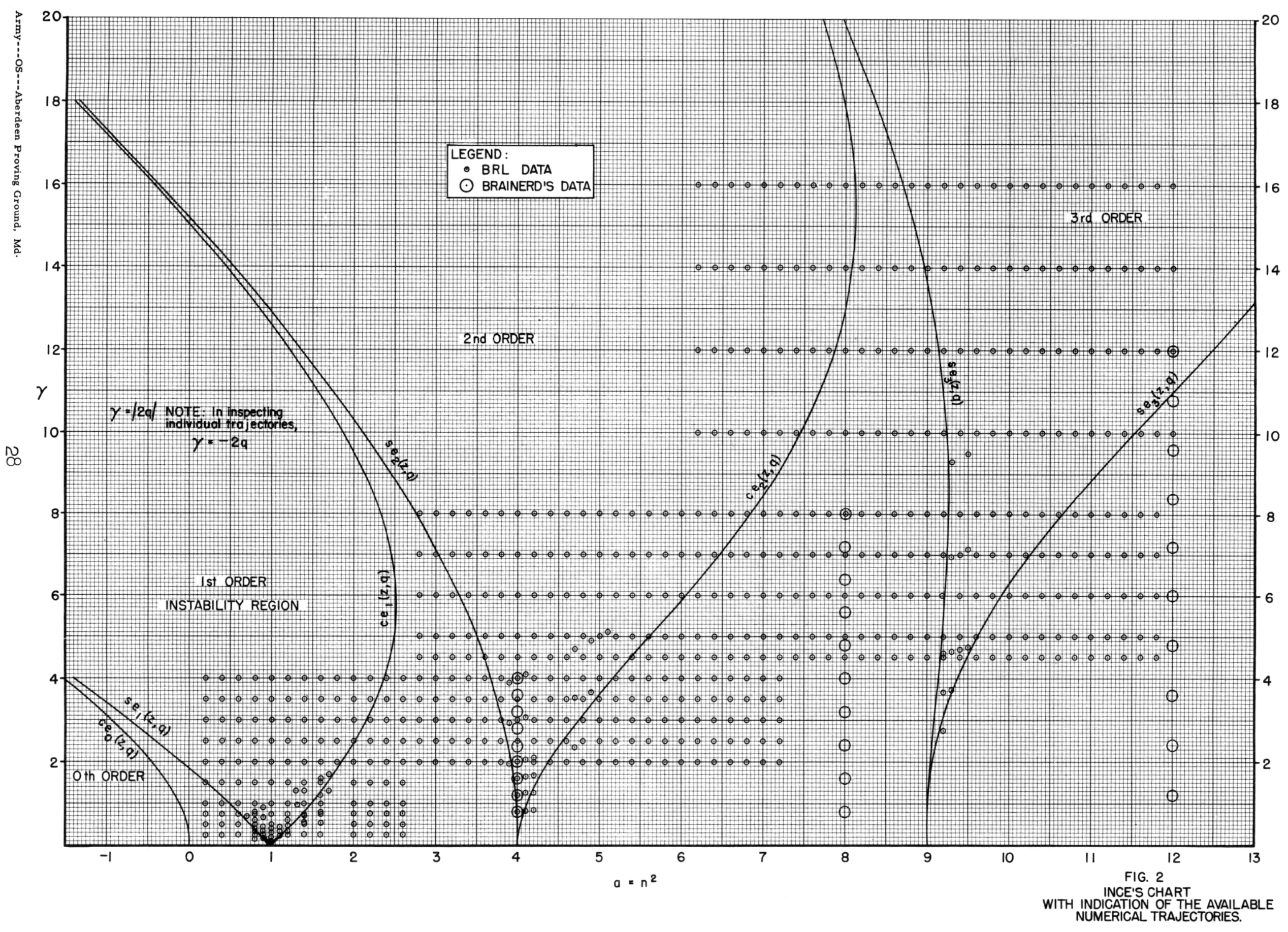




\section{FBFERENCES}

1. N. W. McLachlan, "Theory and Application of Mathieu Functions" (401 pages), Oxford, Cla rendon Press, 1947.

2. W. C. Bickley and N. W. McIachlan, WMathieu Functions of Integral Orders and Their Tabulation", Mathematical Tables and Other Aids to Computation, II, I - 11 (1946-47).

3. L. Brillouin, "A Practical Method of Solving Hill's Eauation", Quarterly of Applied Mathematics, 6, 167-178 (1948).

4. Harry J. Gray, Richard Merwin and J. G. Breinerd, "Solutions of the Mathieu Equation", Transaction of American Institute of Electrical Engineers, 67 (Part I), 429 - 44 I (1948).

5. Unpublished work at the Ballistic Research Laboratories

6. E. T. Whittaker and G. N. Watson, "A Course in Modern Analysis", Cambridge University Press, 1946.

7. Gertrude Blanch, a review in MPAC, II, $263-266$ (1946 - 47). 


\section{DISTRIBUTION LIST}

No. of

Copies

4

10

4 Canadian Army Staff

2450 Massachusetts Avenue, N.W. Washington $B_{2} D$. C.

4 Chief, Bureau of Ordnance

Department of the Navy

Washington 25, D. C.

Attn: $\operatorname{Re} 3$

2 Commander

Naval Proving Ground

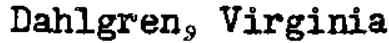

2 Commander

Navy Ordnance Laboratory

White Oak

Silver Spring 19, Maryland

1 Superintendent

Naval Postgraduate School

Monterey, California

2 Commander

Naval Air Missile Test Center

Point Mugu, California

1 Commanding Officer and Director

David W. Taylor Model Basin

Washington $7, D$. C.

1 Commander

Naval Air Development Center

Johnsville, Pennsylvania
2

No. of

Copies

Organization

I Commander

Air Research and Development Command

P. 0. Box 1395

Baltimore 3, Maryland

Director

Air University Library

Maxwell Air Force Base,

Alabama

1 Commander

Arnold Engineering Development Center

Tullahoma, Tennessee

Director

Armed Services Technical

Information Agency

Documents Service Center

Knott Building

Dayton 2, Ohio

4 ASTIA Reference Center

Technical Information Div

Library of Congress

Washington 25, D. C.

National Advisory Committee

for Aerodymamics

1512 H Street, N.W.

Washington $25, D$. C.

Director

National Bureau of Standards

washington 25, D.C.

Attn: Dr. F. L. Alt

Dr. Gertrude Blanch,

Editor, MTAC

1 Commanding Officer

Signal Corps Engineering Laboratories

Fort Monmouth, New Jersey

Attn: Technical Documents Center 
DISTRIBUTION LIST

No. of

Copies

Organization

2 Commanding Officer

Chemical Corps Chemical and Radiological Laboratory

Army Chemical Center, Maryland

Attn: Technical Library

1 Commanding General

Redstone Ârsenal

Huntsville, Alabama

Attn: Technical Library

2 Director

Jet Propulsion Laboratory

Ordnance Corps Installation

Department of the Army

4800 Oak Grove Drive

Pasadena 2, California

Attn: Reports Group

3 California Institute of Technology

Pasadena, California

Attn: Library

1 Massachusetts Institute of Technology

Cambridge 39, Massachusetts

Attn: Library

1 University of Virginia

Charlottesville, Virginia

Attn: Prof. E. J. McShane

I University of Cailifornia

Berkeley, California

Attn: Prof。J。L Kelley

4 University of Pennsylvania

Philadelphia, Pennsylvania

Attn: Library

Dr。J.G. Brainerd

1 Dro I. H. Thomas

Watson Scientific Computing Laboratory

612 West 116th Street

New York 27, New York

1 Professor A. A. Bennett

Department of Mathematics

Brown University

Providence, Rhode Island 


